\title{
Clinical Value of Energy Spectrum Curves of Dual- energy CT in Evaluating Pathological Grading of Gastric Adenocarcinoma
}

\section{Zhihua Lu}

Putian First Hospital of Fujian Province

\section{Suying Wu}

Putian First Hospital of Fujian Province

Jianwei Chen

First Affiliated Hospital of Fujian Medical Univesity

Chuan Yan

First Affiliated Hospital of Fujian Medical University

Yueming Li ( $\nabla$ fjmulym@163.com )

First Affiliated Hospital of Fujian Medical University https://orcid.org/0000-0002-3669-568X

\section{Research article}

Keywords: computed tomography; dual energy; energy spectrum curve; gastric adenocarcinoma; pathology

Posted Date: August 14th, 2019

DOI: https://doi.org/10.21203/rs.2.12786/v1

License: (c) (i) This work is licensed under a Creative Commons Attribution 4.0 International License. Read Full License 


\section{Abstract}

Backgroud: Accurate diagnosis of cancer staging and pathological differentiation are critical for the formulation of individualized treatment and prognosis of gastric cancer. It is vital to explore non-invasive preoperative imaging techniques to evaluate the pathological differentiation degree of gastric cancer tissues, and provide better diagnostic basis and decision-making reference for treatment. The purpose of this study was to explore the clinical value of energy spectrum curves of dual-source dual-energy CT in the quantitative evaluation of different pathological grades of gastric adenocarcinoma. Methods: A total of 62 patients with 1 well, 25 moderately and 36 poorly differentiated gastric adenocarcinomas pathologically confirmed by surgery were collected, and they underwent dual-source dual-energy CT plain scanning and enhanced scanning before operation. Dual-Energy software was used to measure the slope of the energy spectrum curves $(\lambda)$ in arterial and venous phases after image reconstruction. Patients were divided into two groups according to the pathological results, including well and moderately differentiated gastric adenocarcinoma group and poorly differentiated gastric adenocarcinoma group. Data of each group were analyzed by independent sample t-test. The receiver operating characteristic curve was plotted to evaluate the diagnostic efficiency of the corresponding parameters. Results: There were significant differences in $\lambda$ values of $40-50 \mathrm{keV}, 40-60 \mathrm{keV}, 40-80 \mathrm{keV}, 40-90 \mathrm{keV}, 40-100 \mathrm{keV}, 40-120 \mathrm{keV}$, 40-130keV, 40-140keV and 40-150keV energy ranges in venous phase between the well and moderately differentiated group and poorly differentiated group $(P<0.05)$, but no significant differences in $\lambda$ values of different energy ranges in arterial phase between the two groups $(P>0.05)$. And the area under curve in $40-$ $120 \mathrm{keV}$ energy range was the largest in venous phase. $\mathrm{K} 40-120 \mathrm{keV}=2.69$ was selected as the diagnostic threshold with the maximum Youden index, the sensitivity and specificity were $61.1 \%$ and $76 \%$, respectively. Conclusion: The energy spectrum curve of dual-energy CT had certain diagnostic value in the quantitative evaluation of pathological grading of gastric adenocarcinoma.

\section{Introduction}

Gastric cancer is the most common malignant tumor of digestive tract with the third highest mortality rate among cancer-related mortality rates [1]. There are about 1 million new-onset cases of gastric cancer every year. There are regional differences in survival and prognosis of patients with gastric cancer, mainly due to differences in staging criteria. Accurate diagnosis of cancer staging and pathological differentiation is of great significance to the formulation of individualized treatment and prognosis of gastric cancer $[2,3,4]$. The clinical diagnosis of gastric cancer is mainly based on gastroscopy and a definite diagnosis of the pathological differentiation degree of gastric adenocarcinoma mainly depends on postoperative pathological examinations. However, some advanced patients who cannot tolerate surgery and gastroscopy were failed to make a definite diagnosis of the degree of pathological differentiation. Therefore, it is of great clinical significance to actively explore non-invasive preoperative imaging methods to evaluate the pathological differentiation degree of gastric cancer tissues, and to provide more diagnostic basis and decision-making reference for clinical treatment. 
Computer tomography(CT) staging and endoscopic ultrasonography (EUS) staging of gastric cancer were independently classified by the Alliance Against Cancer and the 8th edition American joint committee on cancer (AJCC) TNM staging system $[5,6,7]$. In clinical practice, CT examination has become the main means of $T$ staging of gastric cancer before treatment because of its simplicity, rapidity and objective image advantages [8]. Dual-source CT uses two sets of independent tube detector systems, which can simultaneously obtain the data of substances under high and low energy X-ray, and obtain the attenuation data of substances on X-ray under different energies. Quantitative parameters derived from spectral imaging data on the basis of dual-energy CT have proven to be useful for the diagnosis and staging of gastric cancer $[9,10]$. The purpose of this study was to explore the clinical value of energy spectrum curve of dual-source CT in the quantitative evaluation of gastric adenocarcinoma with different degrees of differentiation.

\section{Materials And Methods}

\section{Patients}

The retrospective study was approved by our Hospital Ethics Committee and the informed consent was waived. 134 Patients with gastric adenocarcinoma pathologically confirmed by surgery in our hospital from May 2018 to September 2018 were collected. 73 patients were included consecutively according to the following inclusion criteria: $\triangle$ Patients undergo surgery after routinely dual-source dual-energy CT

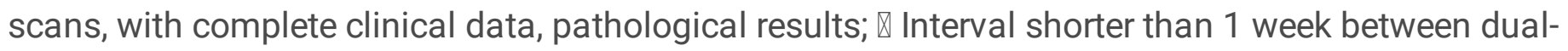
source CT scan and surgery; $\otimes$ No radiotherapy, chemotherapy and other anti-cancer treatments were performed before enhanced CT scanning; $\triangle$ Scanning mode and imaging parameters are in accordance with the unified standard, and iodine contrast agents of the same manufacturer and concentration are used. Of the 73 retrieved patients, 11 were subsequently excluded for the exclusion criteria: खDistant metastasis or no surgical indication; 『Suboptimal image quality for interpretation or image acquired from other institutions; $\bowtie$ Patients with poor gastric filling and poor lesion display. Finally, a total of 62 cases of gastric adenocarcinoma were included, including 46 males and 16 females (range, 33-86 years; mean age, $65.41 \pm 9.73$ years). The patients were divided into three groups according to differentiation status: well, moderately and poorly differentiation. Of the 62 gastric adenocarcinomas, one was highly differentiated adenocarcinoma, 25 were moderately differentiated adenocarcinomas and 36 were poorly differentiated adenocarcinomas (Figure 1).

\section{Dual-source CT scan}

Dual-source dual-energy CT scanner SOMATOM Force was used. Routine plain scanning covering the entire stomach region was performed firstly for each patient in a supine position, followed by three-phase enhanced scanning by using dual-energy mode, including arterial phase, venous phase and delayed phase. During enhanced scanning, a high-pressure syringe was used to inject $370 \mathrm{mg} \mathrm{l} / \mathrm{ml}$ iopromide (1.2 $\mathrm{ml} / \mathrm{kg}$ ), a non-ionic contrast agent, into the antecubital vein (Ultravist, Bayer Healthcare Co., Ltd.) at a rate of $2.5-3.5 \mathrm{ml} / \mathrm{s}$, subsequently $20 \mathrm{ml}$ of saline was injected at the same rate. The automatic trigger mode 
was adopted in dynamic contrast-enhanced scanning, when the CT value reached $100 \mathrm{Hu}$, the arterial phase (AP) scanning was triggered automatically. The venous phase (VP) and delayed phase scanning was performed 60 seconds, 180 seconds respectively after the contrast agent administration. Scanning ranged from the diaphragmatic dome to the inferior pole of kidney and all the images were archived in digital format. Scans were acquired during a single breath-hold with the following parameters: (1)A-tube voltage $100 \mathrm{kV}$, B-tube voltage Sn150 kV; (2)field of view (FOV) 356 mm×356 mm; (3)pitch 0.7; (4)tube rotation time $0.5 \mathrm{~s} / \mathrm{r}$; (5)convolution kernel $\mathrm{Br} 40$; (6)reconstruction section thickness of $1 \mathrm{~mm}$ and slice gap 0mm. Real-time dynamic exposure dose control (Care Dose 4D, Siemens Medical Solutions) technology was used for all data acquisition.

\section{Quantitative Dual-Energy CT Parameters}

Post-processing was performed on the Dual-Energy workstation. An oval or rounded region of interest (ROI) about $0.2-0.4 \mathrm{~cm}^{2}$ drew by one radiologist, avoiding necrosis, calcification, blood vessel as much as possible, was traced in the most obvious enhancement area of the largest diameter of the tumor lesions on a single axial image. After the ROI was traced, the system automatically generated a scatter plot and a spectral curve. The following information was extracted from each lesion and sent for statistical analysis: $\mathrm{HU}$ (Hounsfield unit) at each $\mathrm{x}$-ray beam value between $40 \mathrm{keV}$ and $150 \mathrm{keV}$ (with steps of 10keV).

The slope of the spectral Hounsfield unit curve ( $\lambda$, in Hounsfield unit per kiloelectron-volt), which is defined as the difference between the CT value at two different energy divided by the energy difference, was calculated. For instance, as follows:

\section{$\lambda_{40-150 \mathrm{keV}}=\left(\mathrm{HU}_{40 \mathrm{keV}}-\mathrm{HU}_{150 \mathrm{keV}}\right) /(150 \mathrm{keV}-40 \mathrm{keV})$}

where $\mathrm{HU}_{40 \mathrm{keV}}$ represents the $\mathrm{CT}$ value measured on $40 \mathrm{keV}$ images and $\mathrm{HU}_{150 \mathrm{keV}}$ stands for the $\mathrm{CT}$ value measured on $150 \mathrm{keV}$ images. Totally, $11 \lambda$ values were acquired from a spectrum curve, details are provided in Table 1 and Table 2. $\lambda$ values were measured on both arterial and venous phase contrastenhanced images.

\section{Statistical analysis}

SPSS 22.0 software was used for statistical analysis. According to the pathological results, the patients were divided into two groups, including well and moderately differentiated gastric adenocarcinoma group and poorly differentiated gastric adenocarcinoma group. Measurement data were expressed by mean \pm standard deviation. For data conforming to normal distribution, t-test was used for homogeneous variance; corrected t-test was used for heterogeneity of variance. The receiver operating characteristic 
(ROC) curve was drawn, the area under curve (AUC) was calculated, and diagnostic efficiency of each index was analyzed. $\mathrm{P}<0.05$ was considered as significant difference.

\section{Results}

\section{Clinical data}

Of the 62 patients, there were 26 cases of well and moderately differentiated gastric adenocarcinomas, including 22 males with an average age of $67.33 \pm 7.26$ years, and 4 females with an average age of $65.50 \pm 13.92$ years; there were 36 cases of poorly differentiated adenocarcinoma, including 24 males with an average age of $64.88 \pm 8.59$ years, and 12 females with an average age of $63.08 \pm 14.11$ years. There were no significant differences in age and gender between the two groups.

\section{Comparison of $\lambda$ values between the well and moderately differentiated group and poorly differentiated group}

According to observation and comparison, it can be seen that energy spectrum curves of all gastric adenocarcinomas in arterial phases (Figure 2) and venous phases (Figure 3) were descending. The curves in $40 \mathrm{keV}-110 \mathrm{keV}$ energy range varied greatly and those in $110 \mathrm{keV}-190 \mathrm{keV}$ energy range tended to be flat in arterial and venous phases. (Figure 4).

The $\lambda$ values of poorly differentiated gastric adenocarcinoma group in arterial phase were higher than those of well and moderately differentiated gastric adenocarcinoma group in different energy ranges, but there was no significant difference between the two groups (Table 1). The $\lambda$ values of well and moderately differentiated gastric adenocarcinoma group in venous phase were lower than those of poorly differentiated gastric adenocarcinoma group in different energy ranges: there were significant differences in $\lambda$ values of $40-50 \mathrm{keV}, 40-60 \mathrm{keV}, 40-80 \mathrm{keV}, 40-90 \mathrm{keV}, 40-100 \mathrm{keV}, 40-120 \mathrm{keV}, 40-130 \mathrm{keV}, 40-$ $140 \mathrm{keV}$ and $40-150 \mathrm{keV}$ energy ranges $(P<0.05$ for all), but no significant difference in $\lambda$ values of $40-$ $70 \mathrm{keV}$ and $40-110 \mathrm{keV}$ energy ranges (Table 2 ).

\section{ROC curve analysis}

The ROC curves were plotted with the $\lambda$ values of $40-50 \mathrm{keV}, 40-60 \mathrm{keV}, 40-80 \mathrm{keV}, 40-90 \mathrm{keV}, 40-$ $100 \mathrm{keV}, 40-120 \mathrm{keV}, 40-130 \mathrm{keV}, 40-140 \mathrm{keV}$ and $40-150 \mathrm{keV}$ energy ranges in venous phase. The corresponding AUCs were $0.668,0.669,0.633,0.671,0.693,0.667,0.634$ and 0.681 , respectively (Figure 5). The AUC of $\lambda$ value in $40-120 \mathrm{keV}$ energy range was the largest in venous phase. $\lambda_{40-120 \mathrm{keV}}=2.69$ was selected as the diagnostic threshold with the maximum Youden index, the sensitivity and specificity were $61.1 \%$ and $76.0 \%$, respectively. 


\section{Discussion}

As we all known, the clinical diagnosis of gastric cancer is mainly based on gastroscopy, and the degree of pathological differentiation mainly depends on postoperative pathological examinations, but there were differences in biological behavior, chemotherapy sensitivity [11] and neoadjuvant therapy sensitivity among different differentiated types of gastric adenocarcinoma. Therefore, evaluating the differentiated types of gastric adenocarcinoma before operation, chemotherapy or neoadjuvant therapy has certain clinical significance for individualized treatment and precise treatment of patients. In the present study, dual-source CT scanning technology was used to explore the energy spectrum curve of different energy ranges in evaluating the pathological differentiation of gastric adenocarcinoma, which had important practical significance and clinical value in guiding clinical diagnosis, treatment and prognosis evaluation.

Lesions are showed by conventional CT scanning due to the difference of CT values mostly. Although it can clearly show the location, size, shape, density and enhancement characteristics of the lesions, it has limited ability to differentiate and diagnose substances [12]. Dual-source CT scanning can simultaneously obtain the data of substances under high and low energy $\mathrm{X}$-ray, obtain the attenuation data of substances under different energies, and form the energy spectrum curve of substances. The energy spectrum curve is that the $\mathrm{CT}$ value of $\mathrm{ROI}$ varies with the change of photon energy, the average CT value and standard deviation in each energy range from 40 to $190 \mathrm{keV}$ can be obtained on the energy spectrum curve, which reflects the attenuation characteristics and dynamic changes of substances in different energy ranges [13]. Because the energy spectrum curves of different substances show differences, differences of the energy spectrum curves in different tissue sources, characteristics and components can be estimated, which enables the differential diagnosis. It can be inferred that the energy spectrum curve can be used to differentiate the origin of tumors, benign and malignant tumors, and tumors with different differentiation degrees, moreover, the energy spectrum curve can be quantitatively analyzed to provide new diagnostic ideas [14-15].

In the present study, the energy spectrum curve rates of poorly differentiated gastric adenocarcinoma in venous phase were significantly higher than those of well and moderately differentiated gastric adenocarcinoma in 40-50keV, 40-60keV, 40-80keV, 40-90keV, 40-100keV, 40-120keV, 40-130keV, 40$140 \mathrm{keV}$ and $40-150 \mathrm{keV}$ energy ranges, and the diagnostic efficiency was the highest in 40-120keV energy range. Previous studies on blood perfusion in gastric cancer showed that the vascular permeability-surface value of undifferentiated gastric cancer was higher than that of differentiated gastric cancer [16], the lower the degree of differentiation, the higher the blood volume. In this study, the slope of energy spectrum curve of gastric adenocarcinoma lesions with poorer differentiation was higher, and there was a negative correlation, which indirectly proved that with the decrease of pathological differentiation of gastric cancer, the local enhancement of the lesions and the iodine contrast agent of local tumors increased. It further confirmed the feasibility and reliability of the energy spectrum curve of dual-energy CT imaging in evaluating gastric adenocarcinoma with different degrees of differentiation. 
In our study, there were no significant differences in $\lambda$ values of $40-50 \mathrm{keV}, 40-60 \mathrm{keV}, 40-70 \mathrm{keV}, 40-$ $80 \mathrm{keV}, 40-90 \mathrm{keV}, 40-100 \mathrm{keV}, 40-110 \mathrm{keV}, 40-120 \mathrm{keV}, 40-130 \mathrm{keV}, 40-140 \mathrm{keV}, 40-150 \mathrm{keV}$ energy ranges in arterial phase and $40-70 \mathrm{keV}, 40-110 \mathrm{keV}$ energy ranges in venous phase between well and moderately and poorly differentiated gastric adenocarcinoma, which was inconsistent with the results of Tang et al. [17] that the difference of the slope of the spectral curve in 40-70keV energy range between the two gastric adenocarcinoma groups in arterial and venous phases was statistically significant. We believed that the deviation of this result may be due to the use of different machines, scanning schemes and contrast agents, which needed further studies in the future.

There were still some limitations in this study: (1) Selection bias is inevitable due to the retrospective nature of the study $\llbracket a l t h o u g h$ we set strict inclusion and exclusion criteria. (2) This study mainly investigated gastric adenocarcinoma, which can not reflect differences of other pathological types of gastric cancer (such as gastric signet ring cell carcinoma, gastric squamous cell carcinoma, mucinous gastric carcinoma and so on) with moderately and poorly differentiated adenocarcinoma.

In conclusion, the energy spectrum curve of dual-energy CT in different energy ranges had certain clinical significance in evaluating gastric adenocarcinoma with different degrees of differentiation.

\section{Abbreviations}

CT: computer tomography; EUS: endoscopic ultrasonography; AJCC: American joint committee on cancer; AP: arterial phase; VP: venous phase; FOV: field of view; ROI: region of interest; ROC: receiver operating characteristic; AUC: area under curve.

\section{Declarations}

\section{Acknowledgements}

We thank the radiographers at Department of Radiology, Putian First Hospital of Fujian Province for scanning the patients and data collections in this study.

\section{Authors' contributions}

Yueming Li, Zhihua Lu, Chuan Yan and Jianwei Chen contributed to study design, the clinical study, and manuscript preparation. Zhihua Lu, Chuan Yan and Jianwei Chen contributed to the clinical study and experimental testing. Yueming Li and Suying Wu contributed to study design and manuscript editing. All authors have read and approved the final manuscript.

\section{Funding}


This study was supported by Fujian Provincial Health and Family Planning Commission (CN) (Award Number: 2017-CX-27) and Fujian Provincial Department of Science and Technology (CN) (Award Number: 2015J0105).

\section{Availability of data and materials}

Data for the analyses are available from the corresponding author on request.

\section{Ethics approval and consent to participate}

This study was approved by the Clinical Trials Ethics Committee of 1st Affiliated Hospital of Fujian Medical University ([2016]118). Informed consent was not acquired as this was a retrospective study.

\section{Consent for publication}

Not applicable.

\section{Competing interests}

The authors declare that they have no competing interests.

\section{Author details}

1.Department of Radiology, Putian First Hospital of Fujian Province, Putian, Fujian, China. 2.Department of Radiology, First Affiliated Hospital of Fujian Medical University, Fuzhou, Fujian, China.

\section{References}

[1] Ferlay J, Soerjomataram I, Dikshit R, et al. Cancer incidence and mortality worldwide: sources, methods and major patterns in GLOBOCAN 2012 [J]. International journal of cancer, 2015, 136(5): E35986 .

[2] Kim J P, Lee J H, Kim S J, et al. Clinicopathologic characteristics and prognostic factors in 10783 patients with gastric cancer [J]. Gastric cancer: official journal of the International Gastric Cancer Association and the Japanese Gastric Cancer Association, 1998, 1(2): 125-33.

[3] Wang Z, Xu J, Shi Z, et al. Clinicopathologic characteristics and prognostic of gastric cancer in young patients [J]. Scandinavian journal of gastroenterology, 2016, 51(9): 1043-9.

[4] Zu H, Wang H, Li C, et al. Clinicopathologic characteristics and prognostic value of various histological types in advanced gastric cancer [J]. International journal of clinical and experimental pathology, 2014, 
7(9): 5692-700.

[5] Lu J, Zheng C H, Cao L L, et al. The effectiveness of the 8th American Joint Committee on Cancer TNM classification in the prognosis evaluation of gastric cancer patients: A comparative study between the 7th and 8th editions [J]. European journal of surgical oncology: the journal of the European Society of Surgical Oncology and the British Association of Surgical Oncology, 2017, 43(12): 2349-56.

[6] In H, Solsky I, Palis B, et al. Validation of the 8th Edition of the AJCC TNM Staging System for Gastric Cancer using the National Cancer Database [J]. Annals of surgical oncology, 2017, 24(12): 3683-91.

[7] Li Z, Wang Y, Shan F, et al. ypTNM staging after neoadjuvant chemotherapy in the Chinese gastric cancer population: an evaluation on the prognostic value of the AJCC eighth edition cancer staging system [J].Gastric cancer: official journal of the International Gastric Cancer Association and the Japanese Gastric Cancer Association, 2018, 21(6): 977-87.

[8] Yang L, Shi G, Zhou T, et al. Quantification of the lodine Content of Perigastric Adipose Tissue by DualEnergy CT: A Novel Method for Preoperative Diagnosis of T4-Stage Gastric Cancer [J]. PloS one, 2015, 10(9): e0136871.

[9] Li J, Fang M, Wang R, et al. Diagnostic accuracy of dual-energy CT-based nomograms to predict lymph node metastasis in gastric cancer [J]. European radiology, 2018, 28(12): 5241-9.

[10] Pan Z, Pang L, Ding B, et al. Gastric cancer staging with dual energy spectral CT imaging [J]. PloS one, 2013, 8(2): e53651.

[11] Hallinan J T, Venkatesh S K. Gastric carcinoma: imaging diagnosis, staging and assessment of treatment response [J]. Cancer imaging: the official publication of the International Cancer Imaging Society, 2013, 13(212-27.

[12] Lin XZ, Wu ZY, Tao R, et al. Dual energy spectral CT imaging of insulinoma-Value in preoperative diagnosis compared with conventional multi-detector CT [J]. European journal of radiology, 2012, 81(10): 2487-94.

[13] Karcaaltincaba M, Aktas A. Dual-energy CT revisited with multidetector CT: review of principles and clinical applications [J]. Diagnostic and interventional radiology, 2011, 17(3): 181-94.

[14] Yin Q, Zou X, Zai X, et al. Pancreatic ductal adenocarcinoma and chronic mass-forming pancreatitis: Differentiation with dual-energy MDCT in spectral imaging mode [J]. European journal of radiology, 2015, 84(12): 2470-6.

[15] Wei J, Zhao J, Zhang X, et al. Analysis of dual energy spectral CT and pathological grading of clear cell renal cell carcinoma (ccRCC) [J]. PloS one, 2018, 13(5): e0195699. 
[16] Liang P, Ren X C, Gao J B, et al. lodine Concentration in Spectral CT: Assessment of Prognostic Determinants in Patients With Gastric Adenocarcinoma [J]. AJR American journal of roentgenology, 2017, 209(5): 1033-8.

[17] Tang H, Deng K, Zhao Y, et al. [Use of computed tomography gemstone spectral curve in evaluation on histodifferentiation of gastric cancer] [J]. Zhonghua yi xue za zhi, 2014, 94(45): 3571-4.

\section{Tables}

Table 1 Energy spectrum curve rates of well and moderately differentiated and poorly differentiated gastric adenocarcinoma in arterial phase in different energy ranges

\begin{tabular}{lllll}
\hline & WD* and $\mathrm{MD}^{*} \mathrm{GA}^{*}$ & $\mathrm{PD}^{*} \mathrm{GA}^{*}$ & $\mathrm{t}$ & $\mathrm{p}$ \\
\hline $\mathrm{K} 40 / 50$ & $4.0978 \pm 2.01483$ & $4.6807 \pm 1.2686$ & -1.378 & 0.171 \\
$\mathrm{~K} 40 / 60$ & $3.3263 \pm 1.6202$ & $3.7188 \pm 0.9935$ & -1.172 & 0.246 \\
$\mathrm{~K} 40 / 70$ & $2.69443 \pm 1.3323$ & $3.0797 \pm 0.8343$ & -1.389 & 0.17 \\
$\mathrm{~K} 40 / 80$ & $2.3029 \pm 1.1202$ & $2.5988 \pm 0.70188$ & -1.268 & 0.21 \\
$\mathrm{~K} 40 / 90$ & $1.9747 \pm 0.9617$ & $2.231 \pm 0.6105$ & -1.274 & 0.208 \\
$\mathrm{~K} 40 / 100$ & $1.7206 \pm 0.8384$ & $1.9413 \pm 0.5279$ & -1.262 & 0.212 \\
$\mathrm{~K} 40 / 110$ & $1.5227 \pm 0.7440$ & $1.7313 \pm 0.4647$ & -1.348 & 0.183 \\
$\mathrm{~K} 40 / 120$ & $1.3582 \pm 0.6635$ & $1.5521 \pm 0.4166$ & -1.402 & 0.166 \\
$\mathrm{~K} 40 / 130$ & $1.2378 \pm 0.5956$ & $1.4007 \pm 0.3770$ & -1.308 & 0.196 \\
$\mathrm{~K} 40 / 140$ & $1.1175 \pm 0.5481$ & $1.2736 \pm 0.3434$ & -1.368 & 0.177 \\
\hline $\mathrm{K} 40 / 150$ & $1.0115 \pm 0.5023$ & $1.1542 \pm 0.3280$ & -1.343 & 0.184 \\
\hline
\end{tabular}

*WD:well differentiated; MD: moderately differentiated; PD: poorly differentiated;

*GA: gastric adenocarcinoma

Table 2 Energy spectrum curve rates of well moderately differentiated and poorly differentiated gastric adenocarcinoma in venous phase in different energy ranges 


\begin{tabular}{lclcc}
\hline & WD* and $\mathrm{MD}^{*} \mathrm{GA}^{*}$ & $\mathrm{PD}^{*} \mathrm{GA}^{*}$ & $\mathrm{t}$ & $\mathrm{p}$ \\
\hline $\mathrm{K} 40 / 50$ & $7.466 \pm 1.9516$ & $8.9568 \pm 2.2822$ & -2.658 & 0.01 \\
$\mathrm{~K} 40 / 60$ & $5.9964 \pm 1.5829$ & $7.1988 \pm 1.8393$ & -2.655 & 0.01 \\
$\mathrm{~K} 40 / 70$ & $4.900 \pm 1.3037$ & $5.6482 \pm 1.6540$ & -1.889 & 0.064 \\
$\mathrm{~K} 40 / 80$ & $4.1523 \pm 1.0860$ & $4.9932 \pm 1.2838$ & -2.675 & 0.01 \\
$\mathrm{~K} 40 / 90$ & $3.5598 \pm 0.9392$ & $4.1900 \pm 1.1579$ & -2.34 & 0.023 \\
$\mathrm{~K} 40 / 100$ & $3.1077 \pm 0.8229$ & $3.7401 \pm 0.9581$ & -2.682 & 0.009 \\
$\mathrm{~K} 40 / 110$ & $2.7370 \pm 0.7368$ & $3.1490 \pm 0.9494$ & -1.905 & 0.062 \\
$\mathrm{~K} 40 / 120$ & $2.4587 \pm 0.6541$ & $3.0527 \pm 0.8234$ & -3.006 & 0.004 \\
\hline $\mathrm{K} 40 / 130$ & $2.2281 \pm 0.6046$ & $2.6726 \pm 0.6860$ & -2.61 & 0.011 \\
$\mathrm{~K} 40 / 140$ & $2.0236 \pm 0.5393$ & $2.3792 \pm 0.6439$ & -2.263 & 0.027 \\
\hline $\mathrm{K} 40 / 150$ & $1.8364 \pm 0.5055$ & $2.2391 \pm 0.5824$ & -2.8 & 0.007 \\
\hline
\end{tabular}

*WD:well differentiated; MD: moderately differentiated; PD: poorly differentiated;

*GA: gastric adenocarcinoma

Figures 
134 patients with gastric adenocarcinoma pathologically confirmed by surgery in our hospital from May 2018 to September 2018

73 patients met the following criteria:

(1) Undergoing routinely dual-source CT scan before surgery;

(2) Interval shorter than 1 week between dual-source CT scan and surgery;

(3) No radiotherapy, chemotherapy and other anti-cancer treatments were performed before enhanced CT scanning;

(4) Using the same scanning mode, imaging parameters, iodine contrast agents and concentration.

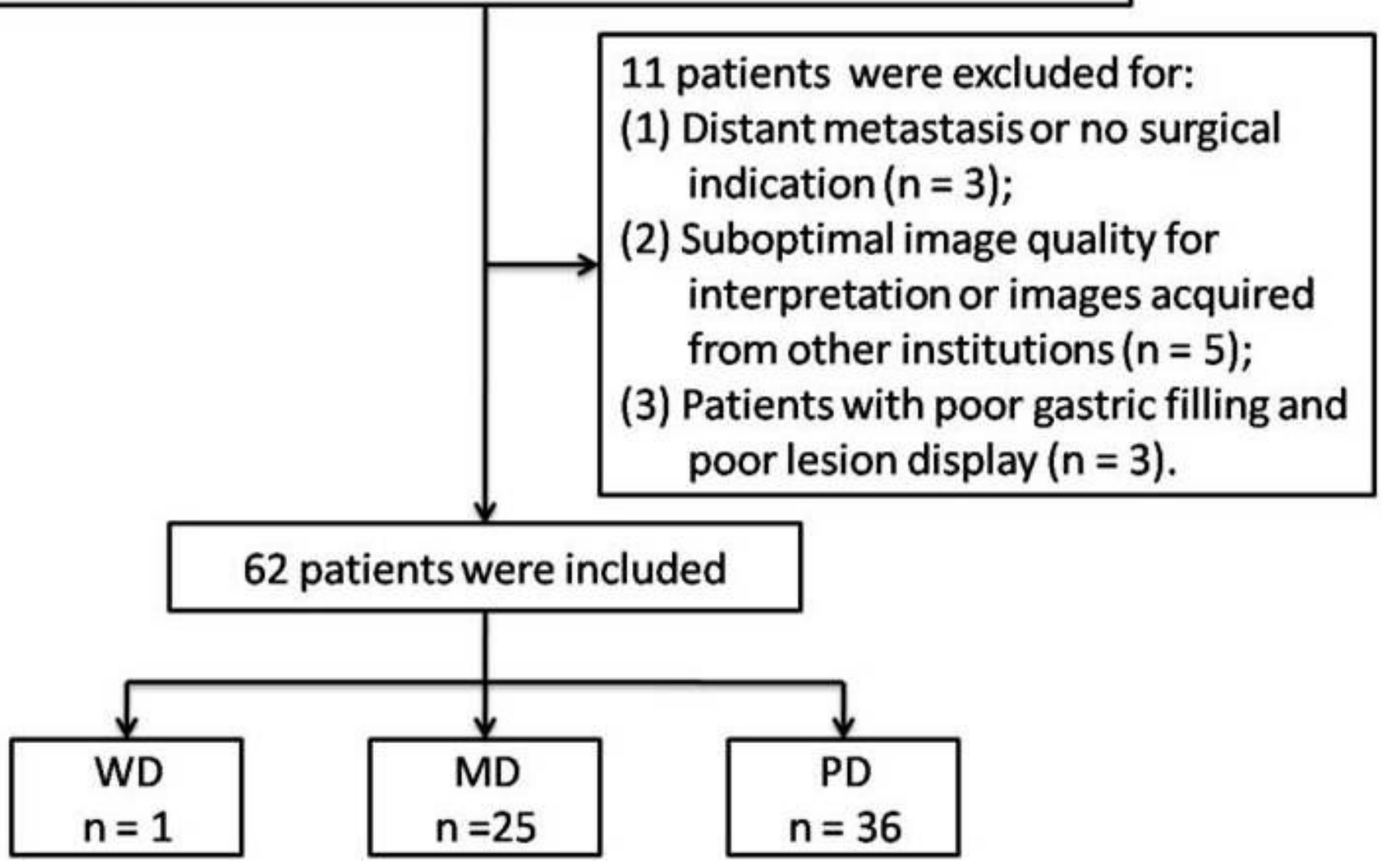

Figure 1

Flow diagram shows inclusion and exclusion criteria for the study. WD = well differentiated, $M D=$ moderately differentiated, $\mathrm{PD}=$ poorly differentiated. 


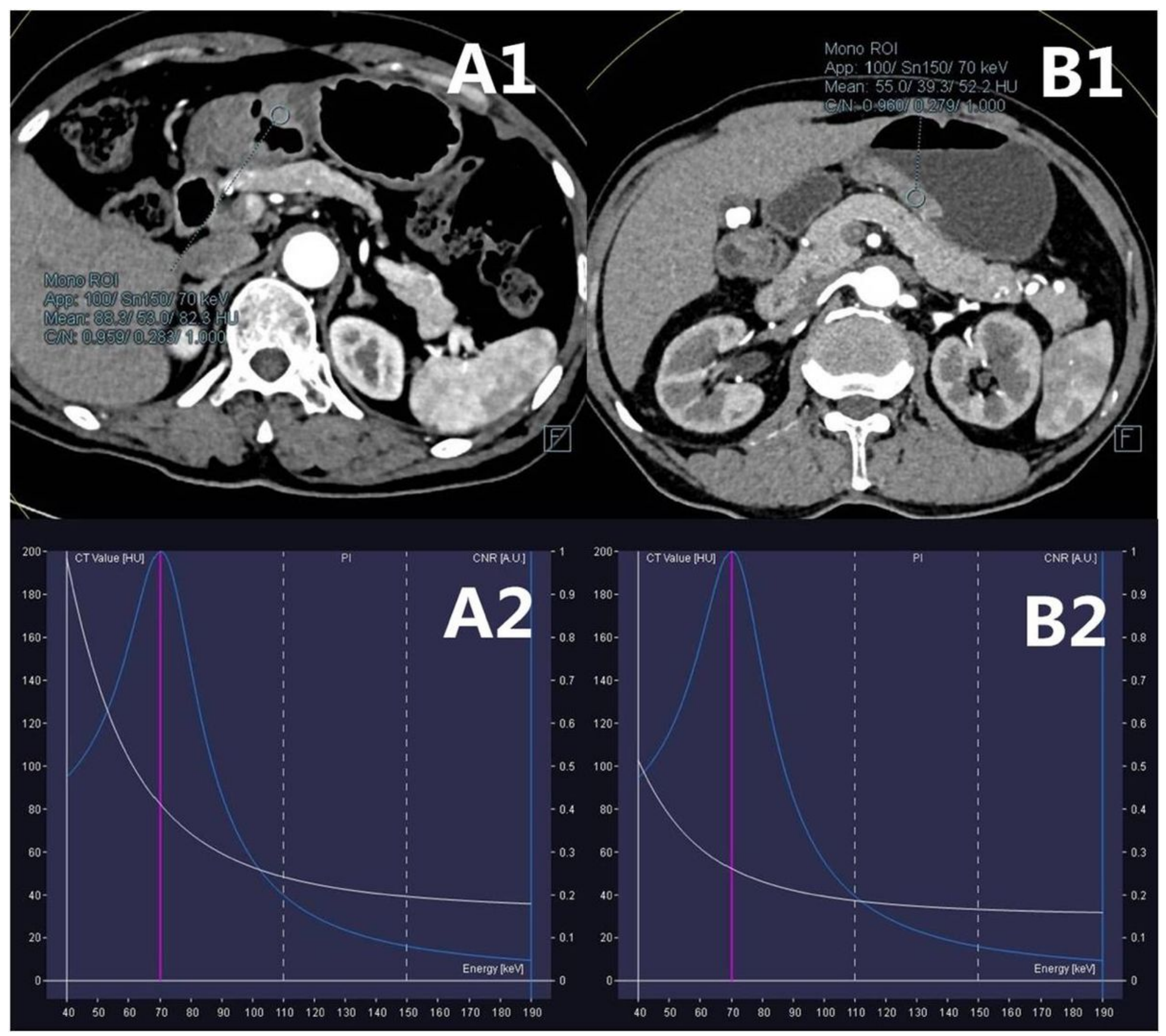

Figure 2

Patient with poorly differentiated gastric adenocarcinoma(A1) and moderately differentiated gastric adenocarcinoma(B1) which show the location of ROI in arterial phase. And the corresponding energy spectrum curves of poorly(A2) and moderately(B2) differentiated gastric adenocarcinoma were displayed. 


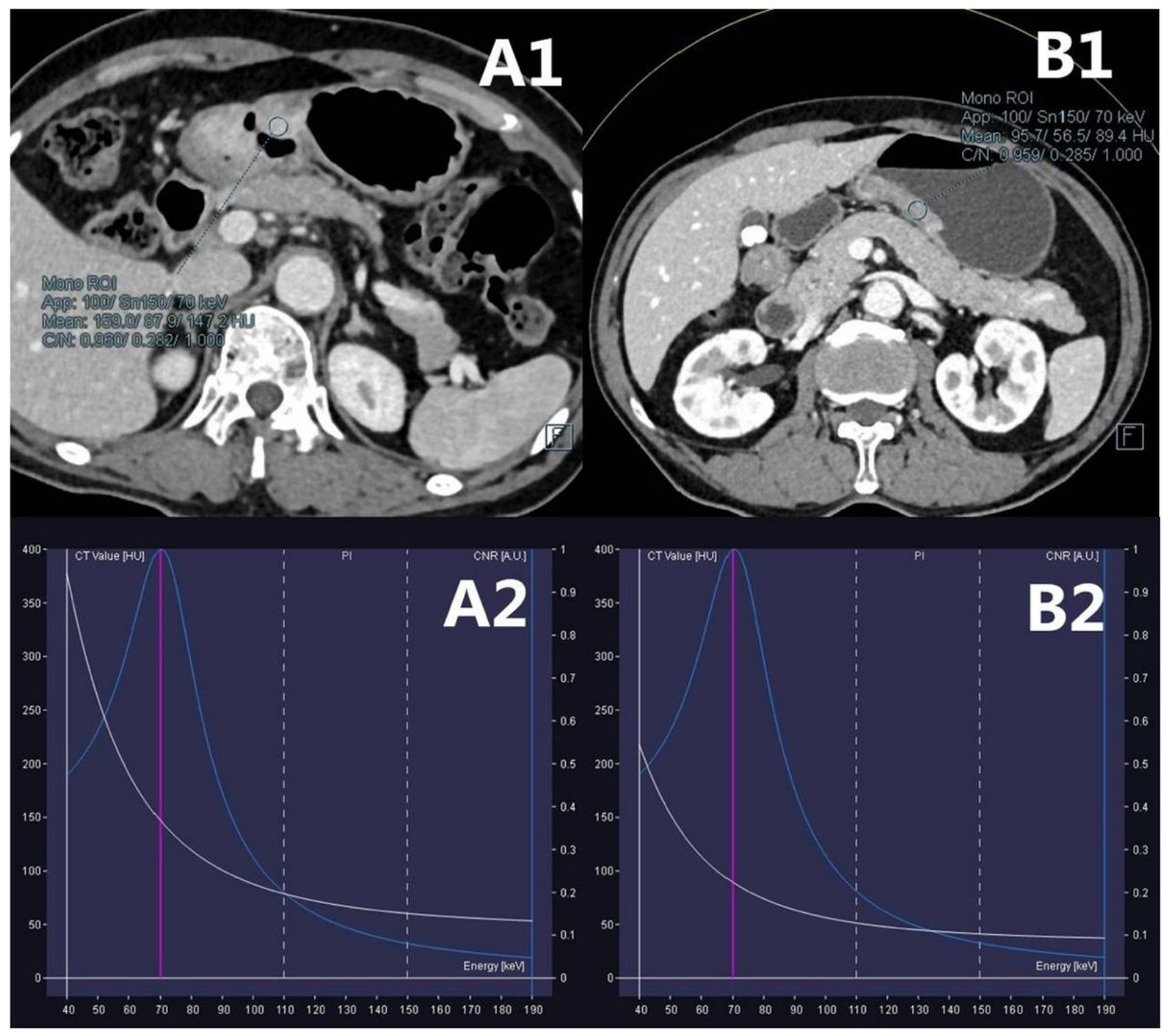

Figure 3

Patient with poorly differentiated gastric adenocarcinoma(A1) and moderately differentiated gastric adenocarcinoma(B1) in venous phase whose location of ROI was same to the arterial phase. And the corresponding energy spectrum curves of poorly(A2) and moderately(B2) differentiated gastric adenocarcinoma were displayed. 


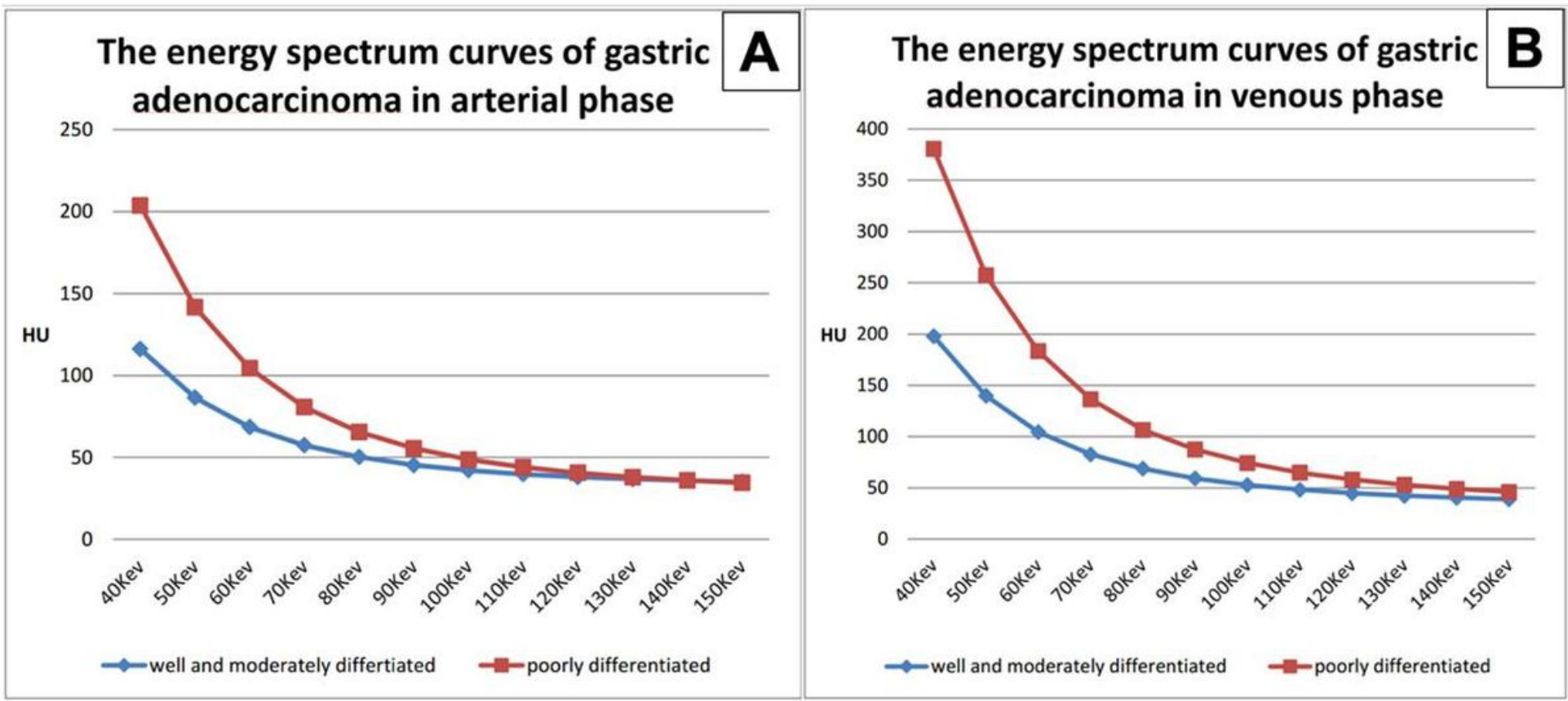

Figure 4

The results showed that the energy spectrum curve of poorly differentiated gastric adenocarcinoma is higher than that of moderately differentiated gastric adenocarcinoma in arterial phase $(A)$ and venous phase(B). 


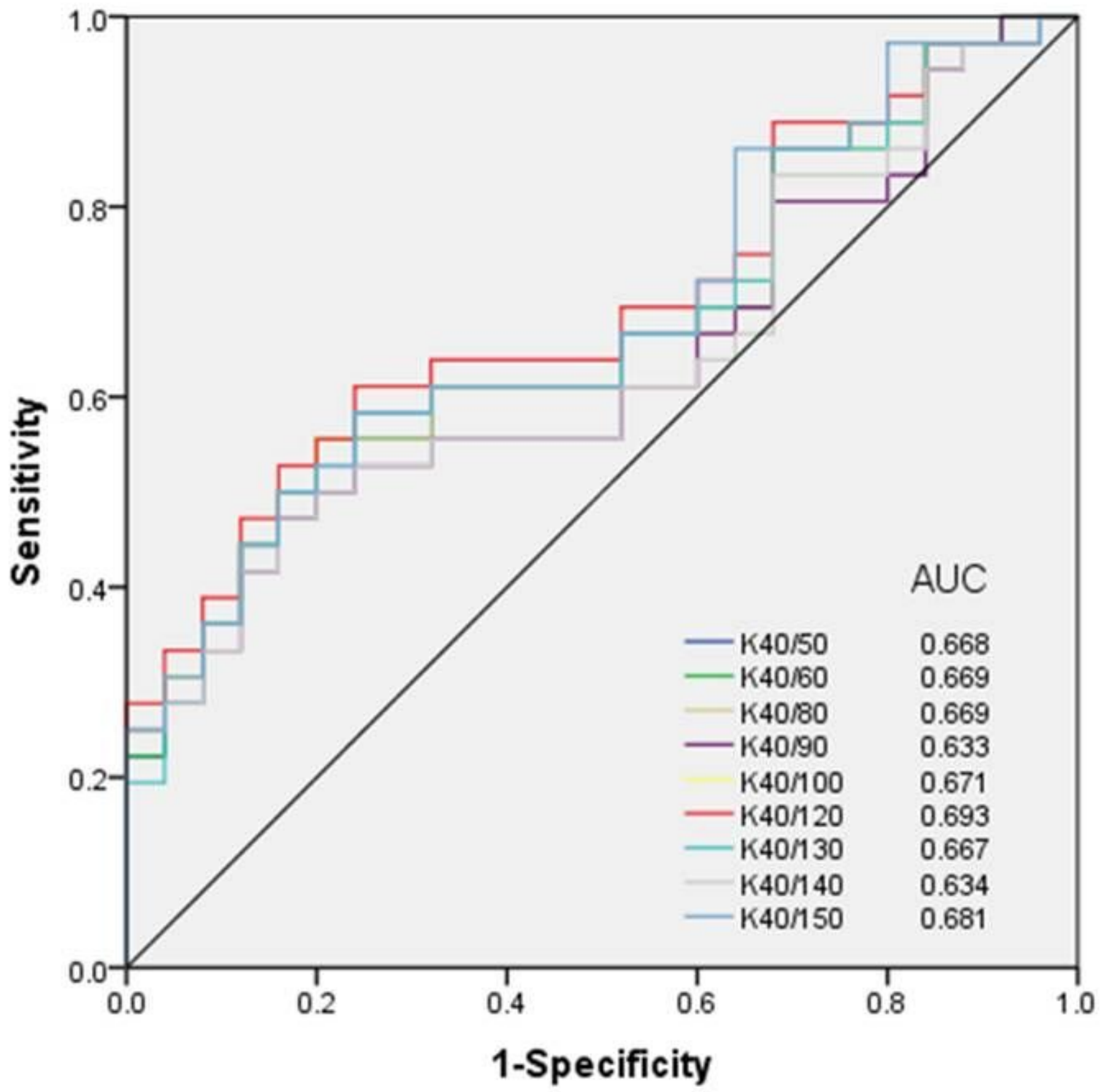

Figure 5

AUCs of ROC curve analysis in different energy ranges in venous phase. The AUC in $\lambda$ of $40-120 \mathrm{keV}$ energy range is largest. 